\title{
Bifurcation of Bingham Streamline Topologies in Rectangular Double-Lid-Driven Cavities
}

\author{
Jianying Zhang \\ Department of Mathematics, Western Washington University, Bellingham, USA \\ Email: Jianying.Zhang@wwu.edu
}

Received September 2014

\begin{abstract}
Numerical simulation of the bifurcation of Bingham fluid streamline topologies in rectangular double-lid-driven cavity, with varying aspect (height to width) ratio $A$, is presented. The lids on the top and bottom move at the same speed but in opposite directions so that symmetric flow patterns are generated. Similar to the Newtonian case, bifurcations occur as the aspect ratio decreases. Special to Bingham fluids, the non-Newtonian indicator, Bingham number $B$, also governs the bifurcation besides the bifurcation parameter $A$.
\end{abstract}

Keywords

Bingham Fluids, Double-Lid-Driven Cavity Flow, Bifurcation, Non-Newtonian Fluids

\section{Introduction}

The lid-driven cavity flow within a rectangular cavity has been studied extensively as a benchmark model not only for testing the validity of numerical methods but also for understanding the rheology of wide varieties of complex industrial fluids. The cavity flow for a single moving lid with varying aspect (height to width) ratio, $A$, generates corner eddies as $A$ increases [1]. Consequently, Sturges [2] considered the symmetric flow with double (the top and bottom) lids moving at the same speed but in opposite directions and revealed the presence of side eddies attached to the stationary walls. For $A \geq 0.9$, the structure of Newtonian fluid flows and a mechanism for eddy generation as $A$ is increased has been fully investigated in [2]. An extension to a more complex class of non-Newtonian fluids, namely Bingham fluids, can be found in [3]. On the other hand, for $A \leq 0.9$, topological changes of the streamlines in Newtonian fluid flows occur as A decreases due to the break or creation of the stagnation points [4]. The main purpose of the present work is to extend the existing results to Bingham fluids, in which the non-Newtonian indicator, Bingham number $B$, plays a significant role in governing the bifurcation.

\section{Constitutive Laws and the Momentum Equations}

Viscoplastic fluids are complex fluids with yield stresses. A viscoplastic fluid behaves like a fluid only when the applied shear stress exceeds the yield stress, otherwise it behaves like a solid. Many multi-component industrial 
fluids are viscoplastic, such as hair gel, mud, cement, paint, processed food and so on. Flow behavior in viscoplastic fluids is a significant and extensively studied topic in processing industry.

Viscoplastic fluids are generalized Newtonian fluids, a class of non-Newtonian fluids. For such fluids, the rate of strain $\dot{\gamma}_{i j}$ and the deviatoric stress $\tau_{i j}$ are related through a constitutive equation of form

$$
\tau_{i j}=\eta(\dot{\gamma}) \dot{\gamma}_{i j} \quad \text { with } \quad \dot{\gamma}=\sqrt{\frac{\dot{\gamma}_{i j} \dot{\gamma}_{i j}}{2}}
$$

where $\eta=\eta(\dot{\gamma})$ is termed the effective viscosity. In the present work, a subscript form such as $M_{i j}$ is always used to indicate a two tensor and its tensor norm is denoted by a non-subscript form such as $M$ defined as $M=\sqrt{\frac{M_{i j} M_{i j}}{2}}$ with the Einstein's notation adopted. Hence the second invariant of the deviatoric stress is denoted by $\tau=\sqrt{\frac{\tau_{i j} \tau_{i j}}{2}}$. If $\tau\left(\dot{\gamma} \rightarrow 0^{+}\right)=\tau_{Y}>0$, then the models are viscoplastic, with yield stress $\tau_{Y}$.

A typical viscoplastic model is the Herschel-Bulkley model with the following scaled constitutive relation:

$$
\tau_{i j}=\left(\dot{\gamma}^{n-1}+\frac{B}{\dot{\gamma}}\right) \dot{\gamma}_{i j} \text { if } \tau>B, \quad \dot{\gamma}=0 \text { if } \tau \leq B .
$$

with $n$ being the power-law index. This is an extension of the power-law model to a fluid with a yield stress, $\hat{\tau}_{Y}$ The dimensionless parameter $B=\frac{\hat{\tau}_{Y} \hat{R}}{\hat{\mu} \hat{V}}$ termed the Bingham number, denotes the ratio of yield stress to viscous stress. Here $\hat{\mu}$ represents the kinematic viscosity, $\hat{R}$ and $\hat{V}$ are the reference spatial and velocity scales, respectively.

For the Herschel-Bulkley model, the effective viscosity is defined from $\tau=\eta(\dot{\gamma}) \dot{\gamma}$. Setting $n=1$ and $B=0$ returns the Newtonian model, $\eta=1$. Setting $n=1$, we recover the popular Bingham model. Note that for the Herschel-Bulkley model, if $B>0$, then $\eta \rightarrow \infty$ as $\dot{\gamma} \rightarrow 0$.

Consider a Bingham fluid in a rectangular cavity $\Omega=[-1,1] \times[-A, A]$, driven by the symmetric sheer motion through the top and bottom lids. The non-dimensionalized momentum equations for the velocity $u=\left(u_{1}, u_{2}\right)$ and the pressure $p$ with the corresponding boundary conditions can be written as

$$
\begin{gathered}
\frac{\partial p}{\partial x_{i}}=\frac{\partial \tau_{i j}}{\partial x_{j}}+g_{i} \text { in } \Omega, \text { for } i=1,2 \\
\nabla \cdot u=0 \text { in } \Omega \\
u_{1}(-1, y)=u_{2}(1, y)=u_{2}(x,-A)=u_{2}(x, A)=0, \quad u_{1}(x,-A)=-1, \quad u_{1}(x, A)=1
\end{gathered}
$$

Where $g=\left(g_{1}, g_{2}\right)=(0,-1)$ is the scaled gravitational acceleration.

Effective numerical algorithm shall be designed and implemented to render the streamlines and yield surfaces in this Bingham cavity flow with desired resolution.

\section{The Augmented Lagrange Method (ALM)}

Theoretically, viscoplastic fluids are generalized Newtonian fluids governed by discontinuous constitutive laws, which implicitly define yield surfaces as interfaces separating the solid and the fluid regions in the corresponding fluids. Due to the unknown shapes and locations of the yield surfaces, the viscous terms in the momentum equations modeling viscoplastic fluid flows cannot be explicitly expressed, which makes the simulation of viscoplastic fluid flows rather difficult. A detailed review and discussion of the existing numerical approaches can be found in [5]. To keep the actual viscoplatic feature of the fluid of interest, we are in favor of the variational approach [6] [7] in the presented work.

The variational reformulation and its application to viscoplastic fluid flows date back to the pioneer work of Duvaut and Lions [8], in which a desired flow motion is captured by solving an equivalent variational inequality whose minimizer set is proven to be the solution set of the momentum equations with the associated constitutive 
law.

It can also be shown via integration by parts that the desired vector field $u$ for the boundary problem (2)-(4) is the one that minimizes

$$
J(v)=\frac{1}{n+1} a(v, v)+j(v)-L(v)
$$

over the admissible set $A$, which is the collection of all the divergence-free $H^{2}(\Omega)$ vector fields satisfying the boundary conditions (4). Here $a(u, v)=\int_{\Omega} \dot{\gamma}^{n-1}(v) \dot{\gamma}_{i j}(u) \dot{\gamma}_{i j}(v)$, referred to as the viscous dissipation rate in some of the literature, is linear in its argument $v$ for general Herschel-Bulkley fluids and bilinear in either of its argument for Bingham fluids, i.e., when $n=1$. The force term $L(v)=-\int_{\Omega} g_{i} v_{i}$ is linear in its argument, whereas the yield stress dissipation rate $j(v)=B \int_{\Omega} \dot{\gamma}(v)$ is nonlinear and non-differentiable in its argument.

The nonlinear and non-differentiable yield stress term is brought in by the discontinuity of the constitutive law of a viscoplastic fluid. The augmented Lagrange method [6] [7], as an effective numerical technique, resolves this difficulty by introducing an auxiliary variable to relax the undesired yield stress term and then adding an augmented constraint. Consequently, the original problem is decoupled into a series of element-wise optimization problems, each of which can be solved with standard optimization techniques. This is also the virtue of the augmented Lagrange method (ALM).

The ALM is implemented based on the variational Equality (5) following the Uzawa type iterations [9]:

- Step 1: Solve an elliptic problem for the velocity. The finite element method is naturally preferred.

- Step 2: Update the pressure based on the incompressible constraint.

- Step 3: Solve element-wise optimization problems for the rate of strain tensor.

- Step 4: Update the Lagrange multiplier corresponding to the augmented constraint.

The detailed numerical implementation of this algorithm can be found in [5] or [9].

\section{Numerical Results}

In the Newtonian case $(B=0)$, the flow pattern starts with a single center at the center of the cavity when $A$ is bigger than 0.318 . Then the center changes to a saddle and breaks down to two centers at the critical value $A=$ 0.318 . This is referred to as the level 1 bifurcation here. When $A$ decreases to 0.169 , the saddle becomes a center, and the two centers become saddles, then two more centers generate. This is referred to as the level 2 bifurcation here. The flow pattern keeps changing as $A$ decreases [4].

Numerical simulation on Bingham fluids with various Bingham number $B$ is conducted. Similar bifurcation process is observed as $A$ drops. However, the yield stress effect of Bingham fluids slows down the bifurcation process. The larger $B$ is (hence the more non-Newtonian the fluid is), the smaller $A$ is required for the streamline to bifurcate. $A$ comparison of the bifurcation status in various $B$ values with the Newtonian case is shown in Table 1 and Table 2, for $A=0.25$ and $A=0.15$, where the Newtonian fluid is undergo the level 1 and level 2 bifurcations, respectively.

Also unique is the existence of the unyielded regions near the left and right walls in Bingham fluids. They tend to squeeze the flow pattern inward as $B$ increases. The $B$ dependence of center locations for $A=0.25$ are shown in Table 3.

Table 1. $B$ dependence of bifurcation when $A=0.25$.

\begin{tabular}{cccc}
\hline & Saddle & Center & Bifurcation level \\
\hline$B=0$ (Newtonian) & 1 & 2 & 1 \\
$B=0.2$ & 1 & 2 & 1 \\
$B=0.5$ & 1 & 2 & 0 \\
$B=1$ & 0 & 1 & 0 \\
$B=5$ & 0 & 1 & 0 \\
\hline
\end{tabular}


Table 2. $B$ dependence of bifurcation when $A=0.15$.

\begin{tabular}{cccc}
\hline & Saddle & Center & Bifurcation level \\
\hline$B=0$ (Newtonian) & 2 & 3 & 2 \\
$B=0.2$ & 2 & 3 & 2 \\
$B=0.3$ & 1 & 2 & 1 \\
$B=2$ & 1 & 2 & 1 \\
$B=5$ & 0 & 1 & 0 \\
\hline
\end{tabular}

Table 3. $B$ dependence of the center locations when $A=0.25$.

\begin{tabular}{cccc}
\hline & Saddle & Center & Bifurcation level \\
\hline$B=0$ (Newtonian) & $(0,0)$ & $( \pm 0.315,0)$ & 1 \\
$B=0.2$ & $(0,0)$ & $( \pm 0.29,0)$ & 1 \\
$B=0.5$ & $(0,0)$ & $( \pm 0.24,0)$ & 1 \\
\hline
\end{tabular}

\section{Conclusion and Future Investigations}

We investigate the bifurcation of Bingham fluid flows in rectangular double-lid-driven cavities with varying aspect ratio $A$ less than 0.9. The proposed numerical algorithm based on an augmented Lagrange approach with a mesh adaptive strategy is implemented to render the streamlines and yield surfaces with desired resolution. Due to the non-Newtonian feature of Bingham fluids, the bifurcation is governed by not only the bifurcation parameter $A$ but the Bingham number $B$ as well. The numerical results motivate the bifurcation analysis based on $B$, which will be studied in the future.

\section{Acknowledgements}

The author would like to thank Western Washington University for the Summer Research Grant awarded in the summer quarter 2014, during which this work was mainly conducted.

\section{References}

[1] Joseph, D.D. and Sturges, L. (1978) The Convergence of Biorthogonal Series for Biharmonic and Stokes Flow Edge Problems: Part II. SIAM Journal on Applied Mathematics, 34, 7-27. http://dx.doi.org/10.1137/0134002

[2] Sturges, L. (1986) Stokes Flow in a Two-Dimensional Cavity with Moving End Walls. Physics of Fluids, 29, 17311734. http://dx.doi.org/10.1063/1.866008

[3] Zhang, J. (2013) Streamline Topologies and Yield Surfaces in Bingham Fluid Flow within Lid-Driven Cavities. Proceedings of the International Conference on Advances in Physics and Engineering Mathematics (ICAPEM), Shanghai.

[4] Gürcan, F. (2003) Streamline Topologies in Stokes Flow within Lid-Driven Cavities. Theoretical and Computational Fluid Dynamics, 17, 19-30. http://dx.doi.org/10.1007/s00162-003-0095-Z

[5] Zhang, J. (2012) An Augmented Lagrangian Approach to Simulating Yield Stress Fluid Flows around a Spherical Gas Bubble. International Journal for Numerical Methods in Fluids, 69, 731-746. http://dx.doi.org/10.1002/fld.2611

[6] Fortin, M. and Glowinski, R. (1983) Augmented Lagrangian Methods: Applications to the Numerical Solution of Boundary-Value Problems. North-Holland, Amsterdam.

[7] Glowinski, R. (1984) Numerical Methods for Nonlinear Variational Problem. Springer-Verlag, New York. http://dx.doi.org/10.1007/978-3-662-12613-4

[8] Duvaut, G. and Lions, J.L. (1976) Inequalities in Mechanics and Physics. Springer-Verlag, Berlin. http://dx.doi.org/10.1007/978-3-642-66165-5

[9] Latché, J.-C. and Vola, D. (2004) Analysis of the Brezzi-Pitkäranta Stabilized Galerkin Scheme for Creeping Flows of Bingham Fluids. SIAM Journal on Numerical Analysis, 42, 1208-1225. http://dx.doi.org/10.1137/S0036142903424386 